\title{
El maestro como gestor de proyectos e investigador social
}

\section{Teacher as a Project Agent and a Social Researcher}

\author{
Nubia Constanza Arias Arias*
}

Fecha de recepción: 23 de febrero de 2009

Fecha de aprobación: 24 de marzo de 20089

\section{Resumen}

En la sociedad actual pensar en la profesión docente implica identificar con claridad el papel que cumple el profesional de la docencia en la construcción de la sociedad; desde esta perspectiva y con las exigencias actuales, el maestro se constituye más que en un ejecutor de procesos programados en un gestor de proyectos de transformación, a partir de los elementos aportados por la investigación social.

En este sentido, es necesario establecer los retos y desafíos que el docente enfrenta a diario y que busca superar con una cualificación constante y el compromiso de hacer cada día su trabajo mejor; esto conlleva a que el maestro se conciba como un investigador social y un gestor de proyectos encaminados a la transformación cultural y la superación de los problemas sociales.

* Investigadora Universidad de San Buenaventura. Correo electrónico: ncariasarias@yahoo.es/ ncarias@hotmail.com. 
Este documento recoge las apreciaciones sobre los aspectos mencionados y le da la opción a los(as) maestros(as) de concebir su trabajo como una oportunidad de transformación social, por tanto, se perciben como líderes en los procesos de análisis de las problemáticas sociales a partir del trabajo pedagógico cotidiano. Se espera que esta visión rescate al docente de la mirada como un simple ejecutor de políticas y lo convierta en un gestor de procesos encaminados al mejoramiento de la calidad de vida de las personas.

Palabras clave: maestro, formación de maestros, gestor de proyectos, investigador social.

\section{Abstract}

In the current society to think about teaching as a profession, implies to identify clearly the teacher's role in the construction of the society. From this perspective and the nowadays requirements, teacher is not a simple doer of programming processes, more than this, and based on elements given by the social research, he/ she becomes an agent of transformation projects.

Thus, it is necessary to identify the challenges and the difficulties that a teacher faces and tries to exceed everyday by being permanent qualified and engaging to perform his/her job better and better. This involves the teacher as a social researcher and projects agent directed towards the cultural transformation and social problems solution.

This document summarizes the opinions about all the aspects mentioned above and gives the teacher the choice to consider his/her job as an opportunity to transform the society by visualizing himself/herself as a leader in the processes analysis about the social problematic, all from his/her daily pedagogical work. It is waited this vision rescues the teacher from a simple achiever of policies and turns him/her into a projects agent improving the quality of life of people.

Key words: teacher, teacher formation, projects agent, social researcher. 


\section{Introducción}

Pensar en el maestro y en su profesión implica develar el papel que cumple éste en el día a día, en la cotidianidad de su trabajo pedagógico; a la vez, esto sugiere presentar los constantes retos a los que se enfrenta en su vida profesional, considerando la necesidad de cualificación constante y el compromiso que tiene por hacer cada día un mejor trabajo para ayudar a superar las crisis de la vida cotidiana de los seres humanos con los que comparte diariamente.

Con los elementos presentados, se puede decir que el maestro, más que un ejecutor de procesos, es un gestor de proyectos y un investigador de los problemas sociales; es por ello que este escrito se centra en argumentar cómo el maestro se puede convertir en un transformador de la cultura y en un líder frente a la reflexión de los problemas sociales que aquejan las comunidades, trabajando más en la formación o proceso de humanización, que en la información, puesto que la formación se constituye en la esperanza para un mañana mejor.

Se espera que esta reflexión sea una invitación a todos(as) los(as) maestros(as) a no desfallecer, a encontrar nuevos sentidos en el trabajo pedagógico, procurando tener la paciencia, la serenidad y la fortaleza para superar las dificultades y encontrar caminos para seguir adelante.

\section{Objetivos}

Reflexionar sobre la concepción del maestro como gestor de proyectos e investigador social, considerando los retos y desafíos que esta concepción representa. Caracterizar al maestro como gestor de proyectos e investigador social, teniendo presente la necesidad de cualificación constante y el compromiso del maestro por hacer mejor su trabajo cada día. Establecer las condiciones para que el maestro sea transformador de la cultura y líder en el análisis de los problemas sociales.

\section{Planteamientos}

\section{Retos y desafios de la profesión docente}

Ser docente significa estar en constante cuestionamiento sobre el trabajo pedagógico diario; implica contribuir a la formación de nuestros estudiantes como personas, más que conocimiento, construir sabiduría (Pérez G., 2004), lo cual les permita asumir a los estudiantes sus propias teorías personales y tomar decisiones para su proyección de vida. Este proceso se constituye en un reto y en un desafío para el maestro, pues cada estudiante tiene unas características tan particulares que contribuir a su formación significa conocer su historia de vida, sus necesidades, fortalezas, sueños y esperanzas; esto hace que cada maestro invierta su diario vivir no sólo en compartir y construir conocimiento, sino también en crear vínculos afectivos y sociales que le permitan al estudiante tener la suficiente confianza para encontrar en el maestro un apoyo para resolver los diferentes problemas de la vida, con la capacidad suficiente de tomar decisiones y proyectarse de la manera más adecuada para su beneficio personal y el de los demás. 
El conocimiento y análisis de la vida de los estudiantes requiere de una actitud interrogativa sobre el contexto en el que se desenvuelven, considerando los problemas que a diario enfrentan las comunidades, lo cual les permite a los estudiantes el desarrollo de un pensamiento crítico y creativo que les brinde la oportunidad de vislumbrar posibilidades para mejorar su calidad de vida y la de los seres que le rodean, desde los conocimientos y las alternativas presentadas por la ciencia y la tecnología; por ello es preciso que cada docente se preocupe por estar informado de los avances científicos y tecnológicos y, en lo posible, encuentre la aplicabilidad de dichos conocimientos en la vida cotidiana, lo que podría facilitar la solución a muchas de las problemáticas que se presentan en el diario vivir de las personas.

En este sentido, el docente ve en cada momento del proceso educativo una oportunidad para el aprendizaje de valores, conocimientos y acciones, esto permite que se genere con sus estudiantes una interacción en la cual el intercambio abra las posibilidades para el encuentro, el respeto y la tolerancia; de esta forma, se generan nuevas maneras de relacionarse, que en muchas ocasiones se constituyen en punto de comparación y reflexión con lo que a diario se vive en la casa, lo cual, a la vez, genera la oportunidad de concebir una vida distinta a la conocida en la convivencia familiar.

En síntesis, podemos afirmar que los retos y los desafíos de la profesión docente se centran en nuestra preocupación constante por seguir preservando el sentido de lo humano, más allá de los avances científicos y tecnológicos, enfa- tizando en su aplicabilidad como contribución al bienestar de todos.

\section{La necesidad de cualificación constante y el compromiso del maestro por hacer cada día un mejor trabajo}

Los que hemos tomado la decisión de ser docentes sabemos que las exigencias de nuestro trabajo diario requieren una cualificación constante, no sólo en el ámbito del conocimiento y de los saberes, sino también y principalmente en relación con lo humano.

Cualificarse en lo humano significa hacer una evaluación constante de la vida y el sentido de la existencia, buscar el equilibrio propio en lo afectivo, lo emocional y la interacción social, teniendo presente que se es ejemplo para los estudiantes y referente de vida para muchos miembros de las comunidades, por ello el compromiso del maestro no es solo consigo mismo, sino que también involucra a las personas con quienes comparte.

En este sentido, es necesario comprender que el compromiso del maestro va más allá de lo temporal, esto se traduce en que marca completamente el estilo de vida de quien se dedica a la profesión docente, lo cual, a su vez, involucra el sentido de la ética y el compromiso social que se asume como maestro y se refleja en el trabajo pedagógico, que se interroga por la situación de crisis que vive la sociedad en la que se está inmerso.

Desde lo planteado se hace necesario considerar el compromiso del maestro por hacer cada 
día su trabajo mejor, teniendo en cuenta las condiciones de los contextos, las particularidades y las necesidades de las personas, pero más que todo la generación de un bienestar para todos y cada uno de los miembros de la comunidad, respetando la diversidad, cumpliendo con el rol asignado como actor social y fuente de cohesión entre los integrantes de la sociedad.

\section{Maestro: más que un ejecutor de procesos, un gestor de proyectos y un investigador de los problemas sociales}

Siguiendo los planteamientos de Donald Schon (1999), se sigue la enseñanza reflexiva o la práctica pedagógica reflexiva; asimismo, se concibe al maestro más que un ejecutor de procesos como un gestor de proyectos y un investigador de los problemas sociales, lo cual implica que el maestro reflexione permanentemente sobre su práctica de enseñanza, con el fin de transformarla hacia una compresión de lo social y el planteamiento de soluciones a las problemáticas de la vida cotidiana, desde la perspectiva de la ciencia y la tecnología; esto genera una estrecha relación entre docencia e investigación, pues evidencia la necesidad del maestro de interrogarse sobre su actuación y crear alternativas de cambio, que es uno de los fines principales de la investigación social desde una perspectiva crítica.

Al tiempo, para que exista relación entre docencia, investigación y proyección social es necesario trabajar desde la perspectiva de la pedagogía crítica; esto se traduce en que el maestro lidere la reflexión sobre las proble- máticas sociales durante el desarrollo de su práctica pedagógica y le presente a los estudiantes la oportunidad de reflexionar y construir alternativas.

Igualmente, en el ámbito comunitario, el maestro puede ser gestor de procesos de transformación creando vínculos con diversos estamentos de la comunidad y estableciendo relaciones para el trabajo colaborativo. Finalmente, lo descrito redunda en apartarse de la mirada programática de ejecutar procesos gestados por otros, en los cuales se desconocen los fines y se reitera en el saber por el saber descontextualizado; esto, a la vez, implica hacer énfasis en los procesos de autogestión y autorregulación que requieren los individuos para proyectar su propia vida y superar las dificultades que se presentan en el día a día.

\section{El maestro transformador de la cultura y líder frente a la reflexión de los problemas sociales}

En la línea de lo expuesto, ser docente es construir cultura, es ser transformador de la realidad con el planteamiento de nuevas visiones y alternativas de solución a las problemáticas de la vida cotidiana; esto permite profundizar en el papel del maestro como actor social y más aún como promotor del cambio, en lo cual la reflexividad es concebida como capacidad de análisis y reflexión continua sobre la realidad, y el proceso de concienciación; hacerse consciente de ser agente de transformación (Freire, 1999); estos aspectos vienen a ser los instrumentos que permiten a los miembros de 
las comunidades trasformar sus realidades y actuar para el mejoramiento de su calidad de vida.

Siendo así, el papel del docente en la sociedad es el de dinamizador de los procesos sociales y culturales, esto implica crear espacios para que los miembros de la sociedad reflexionen sobre sus problemáticas, sus interacciones, sus posibilidades, sus oportunidades y alternativas que ofrecen la ciencia y la tecnología en el mejoramiento de la calidad de vida. En este sentido, el maestro lidera los procesos y propicia las opciones de un trabajo colaborativo entre los miembros de las comunidades.

El maestro es el gestor de los procesos de transformación social, cuando orienta procesos de investigación como la investigación acción participativa. Es un trabajador de la cultura cuando propicia la reflexión sobre la cotidianidad y presenta las opciones para el encuentro y la superación de las dificultades.

\section{Conclusiones}

Al plantear la reflexión sobre la concepción del maestro como gestor de proyectos e investigador social, y considerando los retos y desafíos que esta concepción representa, es fundamental tener en cuenta que el maestro debe superar el modelo academicista y fragmentado del conocimiento disciplinar, lo que implica trabajar sobre la aplicabilidad del conocimiento científico y tecnológico y, a la vez, llevar este conocimiento a un contexto específico, como aporte a la solución de problemas de la cotidianidad.
En este sentido, caracterizar al maestro como gestor de proyectos e investigador social, requiere del rescate de la necesidad de cualificación constante y el compromiso del maestro por hacer mejor su trabajo cada día, esto se traduce en la preocupación del maestro por los problemas de las comunidades y las propuestas de solución a partir del conocimiento construido en el aula.

Con el trabajo del maestro centrado en la construcción del conocimiento contextualizado y la aplicabilidad de los saberes, se pueden dar las condiciones para que el maestro sea transformador de la cultura y líder en el análisis de los problemas sociales, estableciendo las funciones para que los miembros de la comunidad contribuyan al desarrollo y bienestar de todos.

\section{Referencias}

Ayuste, A. et ál. (2001). Planteamientos de la Pedagogía Crítica. Madrid: Paidós.

Díaz, A. \& Espinosa, C. (2001). El docente en las reformas educativas: sujeto o ejecutor de proyectos ajenos. Revista Iberoamericana de Educación, 25.

Freire, P. (1999). Cartas a quien pretende enseñar. México: Fondo de Cultura Económica.

Pérez, A. (2004). La Construcción de Sujeto en la Era Global. En Memorias Encuentro Internacional sobre Políticas, Investigaciones 
y Experiencias en Evaluación Educativa:

consecuencias para la educación. Mayo

12,13,14 y 15 de 2004. Bogotá: UPN-ICFES.

Schon, D. (1999). La formación de profesiona-

les Reflexivos. Madrid: Ediciones Paidós. 